\title{
Consumo no Brasil: Teoria da Renda Permanente, Formação de Hábito e Restrição à Liquidez*
}

\author{
Fábio Augusto Reis Gomes ${ }^{* *}$
}

Sumário: 1. Introdução; 2. TRP, formação de hábito e restrição a liquidez; 3. Base de dados; 4 . Análise do componente cíclico do consumo; 5. Conclusões.

Palavras-chave: consumo; renda; teoria da renda permanente; formação de hábito; restrição à liquidez.

Códigos JEL: E12; E13; E21.

Este artigo analisa a série de consumo agregado do Brasil. Como usual, investiga-se, primeiramente, a aplicabilidade da hipótese do passeio aleatório do consumo, derivada teoricamente a partir das hipóteses de ciclo de vida/renda permanente e expectativas racionais (TRP). Utilizando a decomposição de Beveridge e Nelson (1981) verificamos que o consumo apresenta, além de uma tendência estocástica, uma parte cíclica estacionaria, o que não é compatível com a TRP. Este resultado está em conformidade com o resultado de Reis et alii (1998) de que grande parte da população brasileira está restrita a consumir sua renda corrente, existindo um ciclo comum entre consumo e renda. Em uma tentativa de gerar um processo estocástico para o consumo compatível com a evidência empírica introduzimos formação de hábito nas preferências de um consumidor representativo. No entanto, o processo daí derivado não se mostrou significativo diante da possibilidade dos consumidores serem restritos à liquidez.

This article analyzes the behavior of aggregate consumption series in Brazil. As usual, the random walk hypothesis of consumption, derived theoretically from the life cycle/permanent income and rational expectations hypothesis $(\mathrm{PIH})$, is tested. The Beveridge e Nelson (1981) decomposition indicates that the consumption series has a stochastic trend and a cyclical stationary component, incompatible with the PIH. This result is in line with Reis et alii (1998), who concluded that a large fraction of Brazilian people is

\footnotetext{
${ }^{*}$ Artigo recebido em nov. 2002 e aprovado em out. 2003. O autor agradece os comentários de Afonso Henriques Borges Ferreira, João Victor Issler, Fabio Araujo, Lourenço Senne Paz, Guilherme Hamdan, Heleno Pioner, Humberto Carlos Faria Teixeira, Rodrigo Araújo e as sugestões de dois pareceristas anônimos. Os erros remanescentes são de responsabilidade do autor.

**EPGE/FGV e Centro de Pesquisa em Economia Internacional - CEPE.
} 
credit constrained, which generates a common cycle between consumption and income. Following, we introduce habit formation in the preference of a representative consumer in order to attempt to produce a process of consumption compatible with the empirical evidence. However, such a process does not prove itself statistically significant amidst the possibility of credit constraint consumers.

\section{Introdução}

O objetivo deste artigo é estudar a série temporal de consumo agregado no Brasil, durante o período de 1947 a 1999. Nesta perspectiva, uma primeira análise é feita a partir das hipóteses tradicionais de ciclo de vida/renda permanente e expectativas racionais, abreviadamente TRP (Hall, 1978, Flavin, 1981).

Decorre da TRP que o consumo segue um processo estocástico conhecido como passeio aleatório. Para investigar esta implicação é utilizada a decomposição de Beveridge e Nelson (1981). Esta decomposição é aplicada às séries $\operatorname{ARIMA}(p, 1, q)$, separando-as em parte cíclica e tendência estocástica. Como veremos, Beveridge e Nelson mostraram que a tendência estocástica é um passeio aleatório. Logo, para avaliar a implicação da TRP de que o consumo é um passeio aleatório basta verificar se esta variável não possui um componente cíclico.

Antes de implementar a decomposição de Beveridge e Nelson à série de consumo brasileira é preciso examinar se esta série pode ser representada por um processo ARIMA $(p, 1, q)$. Segue-se que o passo natural consiste em estimar o grau de integração desta série. Nesta análise preliminar, verificamos que as condições necessárias para a aplicação da decomposição de Beveridge e Nelson eram atendidas, uma vez que diagnosticamos o consumo como $I(1){ }^{1}$ Ao utilizarmos esta decomposição, concluímos que a série de consumo agregado brasileira possui um componente cíclico, contrariando a predição da TRP.

O resultado acima é compatível com o trabalho de Reis et alii (1998) segundo o qual existe um ciclo comum entre consumo e renda no Brasil, uma vez que cerca de $80 \%$ da renda no Brasil pertence a consumidores restritos à liquidez. Esses autores obtêm esse resultado através da aplicação da metodologia proposta por Campbell e Mankiw (1989) - que consiste em um modelo heterogêneo, no qual uma fração dos agentes consome segundo a TRP e o restante dos agentes segue a regra de bolso de consumir a renda corrente, possivelmente devido à restrição de liquidez.

\footnotetext{
${ }^{1}$ Este resultado foi obtido inclusive quando consideramos a possibilidade de ocorrer uma mudança estrutural na função de tendência da série de consumo.
} 
Dada a impossibilidade de explicar o consumo agregado no Brasil a partir da TRP, incorporamos a hipótese de formação de hábito em uma tentativa de derivar teoricamente um processo estocástico para o consumo compatível com a evidência empírica. Nossos resultados sugerem que a introdução de formação de hábito constitui-se num procedimento adequado, no sentido de gerar um processo estocástico para o consumo que possui, além da tendência estocástica, um componente cíclico.

Por fim, quando avaliamos a regra de decisão de consumo derivada do modelo com formação de hábito contra a simples regra de bolso de consumir a renda corrente, a regra de bolso demonstrou uma maior eficácia em descrever os dados. ${ }^{2}$ Para chegar a tal conclusão utilizamos, como medida da renda, a série de produto interno bruto do Brasil, de 1947 a 1999.

Em resumo, os resultados encontrados neste artigo sugerem que a série temporal do consumo, no Brasil, é melhor explicada quando se considera um agente que segue a regra de bolso de consumir a sua renda corrente, possivelmente devido à falta de acesso ao crédito.

O artigo está organizado em 4 seções, além desta introdução. Discutimos na seção 2, a TRP, tendo em vista a literatura sobre o caso brasileiro, e apresentamos um modelo com formação de hábito. Na seção 3, é descrita a base de dados. Na seção 4, os resultados dos testes de econométricos são reportados. Por fim, na seção 5, são apresentadas às conclusões do artigo.

\section{TRP, Formação de Hábito e Restrição a Liquidez}

A literatura sobre a decisão de consumo teve grande impulso a partir do trabalho seminal de Hall (1978). O autor demonstrou que sob determinadas condições o consumo segue um passeio aleatório. Pouco tempo depois, Flavin (1981) derivou explicitamente o resultado de Hall (1978) a partir da TRP. ${ }^{3}$ Logo, se a evidência empírica confirmar que o consumo segue um passeio aleatório, teríamos uma evidência a favor da TRP.

\footnotetext{
${ }^{2}$ Weber (2002), utilizando dados para os EUA, também avaliou a performance de um modelo com formação de hábito em relação à regra de bolso de consumir a renda corrente e concluiu que variações na renda não são importantes para explicar variações no consumo, conclusão oposta à encontrada neste artigo. Apesar de motivar seu artigo com uma função utilidade quadrática, Weber (2002) considerou nos testes econométricos outras especificações para as preferências. Aqui, consideramos sempre uma função utilidade quadrática.

${ }^{3}$ Mesmo sendo esses artigos amplamente conhecidos, reproduziremos, ainda que de forma sucinta, a argumentação de Hall (1978) e de Flavin (1981), com o intuito de facilitar discussões posteriores.
} 
Em conformidade com Hall (1978), o problema de otimização intertemporal do consumidor representativo forward-looking pode ser descrito da seguinte forma:

$$
\begin{gathered}
\max _{\left\{c_{t+i}\right\}_{i=0}^{\infty}} E_{t}\left\{\sum_{i=0}^{\infty}\left[\beta^{i} u\left(C_{t+i}\right)\right]\right\} \\
\text { s.a. } A_{t+1+i}=\left(A_{t+i}+Y_{t+i}-C_{t+i}\right)\left(1+r_{t+i}\right) ; \quad A_{0} \text { exógeno }
\end{gathered}
$$

em que $A_{t}, Y_{t}, C_{t}$ e $r_{t}$ são, respectivamente, riqueza, renda, consumo e taxa de juros no período $t$ e $\beta$ é o fator de desconto. Assim, sujeito à restrição orçamentária, o consumidor escolhe o consumo ao longo do tempo, sendo que a solução deste problema deve atender a Equação de Euler (1),

$$
u^{\prime}\left(C_{t}\right)=\beta\left(1+r_{t}\right) E_{t}\left[u^{\prime}\left(C_{t+1}\right)\right]
$$

Hall (1978) pressupõe as seguintes hipóteses: $r_{t}=r, \beta(1+r)=1$ e uma função utilidade quadrática do tipo $u\left(C_{t}\right)=-\frac{a}{2}\left(\bar{C}-C_{t}\right)^{2}$, obtendo daí a hipótese do passeio aleatório do consumo, $C_{t+1}=C_{t}+\epsilon_{t+1}$, em que $\epsilon_{t+1}$ é uma inovação. ${ }^{4}$

A TRP sugere que cada agente consome a cada período sua renda permanente, $Y_{t}^{P}$, que é dada por:

$$
Y_{t}^{P}=r\left[A_{t}+\sum_{i=0}^{\infty}\left(\frac{1}{1+r}\right)^{i+1} E_{t}\left(Y_{l, t+i}\right)\right]
$$

em que $Y_{l, t}$ é a renda do trabalho no período $t$. Assim, a renda permanente pode ser vista como um fluxo de recursos constantes, condicionado à expectativa no período $t$, que pode ser sustentado pelo restante do horizonte de vida do indivíduo, possuindo a propriedade $E_{t}\left(Y_{t+1}^{P}\right)=Y_{t}^{P}$. Como $C_{t}=Y_{t}^{P}$, é trivialmente derivada a hipótese do passeio aleatório do consumo (Flavin, 1981). ${ }^{5}$ Utilizando dados referentes aos EUA, Flavin (1981) rejeitou a TRP devido às evidências encontradas de que o consumo corrente responde a renda corrente em magnitude superior àquela que poderia ser atribuída ao papel da renda corrente em sinalizar variações na renda permanente. Diz-se, então, que existe um excesso de sensibilidade do consumo a renda corrente. ${ }^{6}$

\footnotetext{
${ }^{4}$ Isto equivale a dizer que $\epsilon_{t+1}$ é ortogonal a toda variável que pertence ao conjunto de informação do consumidor em $t, I_{t}$, daí $E_{t}\left(\epsilon_{t+1}\right)=E\left(\epsilon_{t+1} \mid I_{t}\right)=0$.

${ }^{5}$ Vale notar apenas que Flavin (1981), ao contrário de Hall (1978), não utilizou a hipótese de que as preferências são quadráticas para derivar a hipótese do passeio aleatório do consumo.

${ }^{6}$ Flavin (1981) obteve esse resultado ao derivar um modelo econométrico estrutural que inclui a forma reduzida analisada por Hall (1978).
} 
Segundo Campbell e Mankiw, as evidências empíricas para os EUA são melhor explicadas quando os dados são gerados não apenas por um único consumidor, mas por dois tipos de consumidores. O primeiro consumiria sua renda corrente, à la Keynes, o que pode ser devido à falta de acesso ao crédito. $^{7}$ Já o segundo tipo consumiria a sua renda permanente à la Hall (1978). Assim o consumo agregado seria $C_{t}=C_{K t}+C_{H t}$, formando-se o seguinte teste de hipótese: $H_{0}: \Delta C_{t}=\epsilon_{t}$ (tipo Hall) e $H_{1}: \Delta C_{t}=\Delta Y_{t}$ (tipo Keynesiano), que foi implementado pelos autores através da seguinte equação de teste,

$$
\Delta C_{t}=\lambda \Delta Y_{t}+(1-\lambda) \epsilon_{t}
$$

em que $\epsilon_{t}$ é uma inovação e $\lambda$ a proporção da renda que pertence a consumidores que estão restritos a consumir sua renda corrente. A TRP é testada através da hipótese nula de que $\lambda=0$. Neste caso, a equação acima torna-se $\Delta C_{t}=\epsilon_{t}$, ou seja, o consumo segue um passeio aleatório. O que Campbell e Mankiw (1989) não explicitaram é que a equação de teste (2) consiste em um teste de ciclo comum entre consumo e renda, ${ }^{8}$ o que fica claro ao reescrevê-la da seguinte forma:

$$
[1-\lambda]=\left[\begin{array}{c}
\Delta C_{t} \\
\Delta Y_{t}
\end{array}\right]=(1-\lambda) \varepsilon_{t}
$$

Assim, $[1-\lambda]$ é o vetor que combina $\Delta C_{t}$ e $\Delta Y_{t}$ de forma a anular suas correlações seriais, já que $\epsilon_{t}$ é uma inovação. Como Campbell e Mankiw estimaram $\lambda$ aproximadamente igual a meio e estatisticamente diferente de zero, concluímos que consumo e renda possuem um ciclo comum. ${ }^{9}$ Por conseguinte, a TRP não é capaz de replicar satisfatoriamente a evidência empírica para os EUA. Certamente há uma relação entre este resultado e o resultado de Flavin (1981) de excesso de sensibilidade do consumo a renda. Note que, pois se o consumo possui um componente cíclico não considerado devidamente nas equações de teste e este ciclo é comum ao da renda, não é surpreendente que o consumo reaja à renda, quando esta é incluída na equação de teste.

Utilizando a metodologia proposta por Campbell e Mankiw, Reis et alii (1998) estudaram várias séries anuais de consumo agregado do Brasil, datadas de 1947

${ }^{7}$ Este caso ficou conhecido na literatura como keynesiano. Contudo, pode ser coberto pelo modelo neoclássico quando se considera que o indivíduo desconta totalmente o futuro - neste caso o indivíduo é considerado "míope". No entanto, a explicação mais plausível para esse comportamento seria a falta de acesso ao crédito.

${ }^{8}$ Para maiores detalhes sobre ciclo comum, ver Engle e Kozicki (1993).

${ }^{9}$ Esta conclusão se deve também ao fato de que, após a estimação da equação (2) através do método de variáveis instrumentais, não foi rejeitado o teste de sobreidentificação de validade dos instrumentos. 
a 1994, e também rejeitaram a TRP, tendo em vista a estimativa obtida de que aproximadamente $80 \%$ da renda brasileira pertence a consumidores restritos a consumir apenas a sua renda corrente. Esse resultado sugere a existência de um ciclo comum entre o consumo e a renda no Brasil. Portanto, a estimativa de um $\lambda$ significativamente diferente de zero leva a rejeição da TRP. ${ }^{10}$

Uma forma mais direta e geral de investigar a existência de um ciclo no consumo é aplicar a decomposição de Beveridge e Nelson, uma vez que esta decomposição indica se o consumo possui um componente cíclico, independente de este ser comum ou não ao componente cíclico da renda.

Segundo Beveridge e Nelson, toda série, $x_{t}$, que possui uma representação ARIMA $(p, 1, q)$ pode ser decomposta em uma tendência estocástica, $x_{t}^{p}$, e um componente cíclico estacionário, $x_{t}^{c}$, de modo que,

$$
\begin{gathered}
x_{t}=x_{t}^{p}+x_{t}^{c} \\
x_{t}^{p}=x_{t-1}^{p}+\psi_{t}, \quad \psi_{t} \text { é uma inovação } \\
x_{t}^{c} \quad \text { é estacionário }
\end{gathered}
$$

Como Beveridge e Nelson mostraram que a tendência estocástica é sempre um passeio aleatório, inclusive quando existe um componente cíclico, a TRP implica que a decomposição de Beveridge e Nelson do consumo deve possuir apenas a tendência estocástica. Dito de outra forma, o consumo não deve possuir um componente cíclico. Assim uma maneira de interpretar o resultado de Reis et alii (1998) é dizer que a estimativa de $\lambda \neq 0$ implica que o consumo possui um componente cíclico, o que por si só contraria a TRP.

Neste ponto, destacamos que o resultado de Hall (1978) foi derivado a partir do problema de otimização do consumidor e, na medida em que a TRP é rejeitada, este problema de otimização também é colocado em xeque. Assim, caso a decomposição de Beveridge e Nelson indique que existe um ciclo no consumo, rejeita-se a TRP, mas há ainda uma possibilidade de se reformular este problema de otimização, compatibilizando-o com a evidência empírica. Para tanto, é necessário derivar teoricamente, a partir do problema do consumidor, um processo estocástico para o consumo que possua um componente cíclico. Isto pode ser feito se adotarmos preferências que exibam formação de hábito. Esta modificação tem

\footnotetext{
${ }^{10}$ As estimativas obtidas por Reis et alii (1998) não foram rejeitadas nos testes de sobreidentificação, fato crucial para a conclusão de que existe um ciclo comum entre renda e consumo no Brasil.
} 
como apelo os bons resultados obtidos na literatura de finanças com este tipo de preferências e o fato de a pesquisa em psicologia sugerir que a função utilidade deveria incluir algum nível de referência, uma vez que as pessoas são mais sensíveis a mudanças do que aos níveis absolutos (Rabin, 1998).

A intuição de porque a introdução de formação de hábito gera um componente cíclico no consumo decorre da concepção do que é o ciclo. Se o ciclo pode ser entendido como uma sucessão de períodos nos quais a série permanece acima ou abaixo de sua tendência com alguma persistência, a existência de ciclo em uma série está intimamente ligada à existência de inércia na série e a introdução de formação de hábito dá origem justamente a essa inércia na decisão de consumo.

Em conformidade com Weber (2002) ${ }^{11}$, a introdução de formação de hábito no problema de otimização intertemporal do consumidor é feita ao se considerar uma função utilidade do tipo,

$$
u\left(C_{t}, C_{t-1}\right)=-\frac{a}{2}\left[\bar{C}-\left(C_{t}-b C_{t-1}\right)\right]^{2}
$$

em que $b$ representa a intensidade do hábito na função utilidade. Com isso, o problema de otimização intertemporal do consumidor torna-se:

$$
\max _{\left\{c_{t+i}\right\}_{i=0}^{\infty}} E_{t}\left\{\sum_{i=0}^{\infty}\left[\beta^{i} u\left(C_{t+i}, C_{t+i-1}\right)\right]\right\}
$$

s.a. $A_{t+1+i}=\left(A_{t+i}+Y_{t+i}-C_{t+i}\right)\left(1+r_{t+i}\right) ; \quad A_{0}$ e $C_{-1}$ exógenos

Mais uma vez, considerando $r_{t}=r$ e $\beta(1+r)=1$, obtemos o seguinte processo:

$$
C_{t+2}=\left(\frac{1}{b \beta}+b+1\right) C_{t+1}-\left(\frac{1}{b \beta}+b+\frac{1}{\beta}\right) C_{t}+\frac{1}{\beta} C_{t-1}+v_{t+2}
$$

Assim, fica claro que o processo teórico do consumo possui um componente cíclico, além da tendência estocástica, e em principio, é capaz de dar conta da evidência empírica. ${ }^{12}$ Para avaliar esta questão, podemos utilizar a metodologia

\footnotetext{
${ }^{11}$ Weber (2002) introduziu formação de hábito nas preferências para avaliar se o resultado de Campbell e Mankiw, de que cerca de $50 \%$ da renda disponível nos EUA pertence a consumidores restritos a consumir sua renda corrente, resiste à introdução de não separabilidade intertemporal na função utilidade. Weber (2002) conclui que não há evidência a favor de restrição de liquidez para o caso dos EUA.

${ }^{12}$ Como utilizaremos nas equações de teste, mais a frente, instrumentos defasados no mínimo em 2 períodos, a equação acima está bem definida.
} 
de Campbell e Mankiw com uma nova hipótese nula, o modelo com formação de hábito e uma nova equação de teste, de modo que,

$$
\begin{aligned}
H_{0} & : \lambda=0 \\
H_{1} & : 1 \geq \lambda>0 \\
\Rightarrow \Delta C_{t+2} & =\lambda \Delta Y_{t+2}+(1-\lambda)\left(\beta_{1} \Delta C_{t+1}+\beta_{2} \Delta C_{t}\right)+\mu_{t+2}
\end{aligned}
$$

em que $\mu_{t+2}=(1-\lambda) v_{t+2}$.

Portanto, podemos discutir se a existência de um ciclo no consumo se deve a formação de hábito ou ao fato de os agentes estarem restritos a consumir sua renda corrente.

\section{Base de Dados}

As séries brasileiras de consumo agregado, produto interno bruto (PIB) e deflator implícito do PIB utilizadas foram geradas pelo Instituto Brasileiro de Geografia e Estatística - IBGE e foram obtidas no IPEADATA. Estas séries apresentam freqüência anual, cobrindo o período de 1947 a 1999, estando todas na mesma unidade - $\mathrm{R} \$$. Consideramos duas séries de consumo agregado. A primeira série de consumo é composta pelo consumo final das famílias somado à variação de estoques. A justificativa para tal soma reside no fato de que para os anos de 1987 a 1989 não havia dados em separado para estes dois componentes da despesa agregada. A segunda série é composta por dados anuais sobre consumo total ${ }^{13}$, sendo que os anos de 1987 a 1989 incluem variações de estoque. Como medida da renda, foi utilizada a série histórica do PIB.

Foram construídas séries em valor real deflacionando-se as séries nominais pelo deflator implícito do PIB. Além disso, nas equações de teste foram utilizados o logaritmo das séries.

A partir deste ponto, é utilizada a seguinte notação: CF é o logaritmo da soma da série de consumo final das famílias e da série de variação de estoques em valor real (figura A.1 e figura A.4), analogamente CT refere-se a série de consumo total (figura A.2 e figura A.5) e PIB refere-se a série do PIB (figura A.3 e figura A.6). Observando as figuras de CF, CT e PIB, notamos que todas as séries possuem um comportamento bastante semelhante, inclusive apresentando uma tendência que

\footnotetext{
${ }^{13}$ Refere-se ao consumo final das famílias e das administrações públicas, deduzindo-se os pagamentos parciais efetuados pelas famílias.
} 
pode estar sendo gerada por uma raiz unitária. Esta suspeita é corroborada pela análise dos correlogramas (ACF e PACF $),{ }^{14}$ uma vez que para todas as séries a ACF decai lentamente e a PACF apresenta um spike na primeira defasagem.

\section{Análise do Componente Cíclico do Consumo}

\subsection{Decomposição de Beveridge e Nelson}

Antes de investigar a existência de um componente cíclico no consumo, é preciso verificar se as condições necessárias para a aplicação da decomposição de Beveridge e Nelson são satisfeitas. Dito de outra forma, verificaremos se o consumo pode ser representado por um processo $\operatorname{ARIMA}(p, 1, q)$. O primeiro passo consiste na estimação do grau de integração da série de consumo, o que é feito através de testes de raiz unitária, admitindo-se, inclusive, uma mudança estrutural na função de tendência da equação de teste. Caso $C_{t} \sim I(1), \Delta C_{t}$ é estacionária. Pelo teorema de decomposição de Wold, $\Delta C_{t} \sim M A(\infty)$ e pode ser aproximada por um processo $\operatorname{ARMA}(p, q)$. Finalmente, $C_{t} \sim \operatorname{ARIMA}(p, 1, q)$, podendo ser aplicada a decomposição de Beveridge e Nelson. ${ }^{15}$ Além disso, como Beveridge e Nelson mostraram que a tendência estocástica é um passeio aleatório, inclusive quando há um componente cíclico, a TRP implica que o consumo deve possuir apenas esta tendência. Em outras palavras, a TRP implica que o consumo deve possuir uma raiz unitária e um componente cíclico nulo $p=q=0$.

A análise do grau de integração do consumo é feita através do teste de hipótese aumentado de Dickey-Fuller (ADF), que é baseado na estimação da seguinte equação de teste,

$$
\Delta C_{t}=\mu+\beta t+\delta C_{t-1}+\sum_{i=1}^{n} \lambda_{i} \Delta C_{t-i}+e_{t}
$$

em que:

$\Delta$ é o operador de diferença;

$\mu$ é uma constante; e

$t$ é uma tendência linear.

\footnotetext{
${ }^{14} \mathrm{ACF}$ refere-se à função de autocorrelação e PACF a função de autocorrelação parcial.

${ }^{15}$ Note-se que o fato de $C_{t} \sim \operatorname{ARIMA}(p, 1, q)$ não impede que este seja um passeio aleatório, como predito pela TRP. Para tanto basta que $p=q=0$. Se isto ocorre, o consumo não possui um componente cíclico.
} 
A especificação da equação (4) foi determinada com base na metodologia proposta por Holden e Perman (1994) que visa avaliar a significância estatística da constante e da tendência linear. ${ }^{16}$ A especificação selecionada tanto para CF quanto para CT não inclui a tendência linear. Utilizando então o teste ADF não rejeitamos a hipótese nula de que estas séries apresentam uma raiz unitária, no nível de significância de $5 \%$ (tabelas A1 e A2). ${ }^{17}$ Este resultado está em conformidade com aqueles obtidos por Rocha e Issler (2000) e Reis et alii (1998).

O diagnóstico acima indica a existência de uma raiz unitária na série de CF e CT. Porém muito tem se discutido sobre a validade de testes como estes quando aplicados a séries que possuem uma mudança estrutural (Perron, 1989, 1997). Ademais, segundo Cati (1998), é possível rejeitar a hipótese nula de raiz unitária para a série do PIB brasileiro de 1947 a 1993, se permitimos uma mudança na inclinação da sua função de tendência em 1980 ou 1985, dependendo do método utilizado para determinar o ponto de quebra, e, como é bem sabido, esta variável é o principal argumento da função consumo. Assim, utilizaremos uma equação que estime o grau de integração da série de CF e CT, levando em conta uma possível mudança estrutural nestas séries.

Implementamos, então, a metodologia proposta por Perron $(1989,1997)$ que possibilita a ocorrência de uma mudança estrutural no comportamento de CF e CT, tanto sob a hipótese nula quanto sob a hipótese alternativa. A equação de teste proposta por Perron (1989) é a seguinte:

$$
C_{t}=\mu+\theta D U_{t}+\beta t+\gamma D T_{t}+d D(T B)_{t}+\alpha C_{t-1}+\sum_{i=1}^{k} \omega_{i} \Delta C_{t-i}+\epsilon_{t}
$$

em que $D(T B)_{t}=1$ se $t=T_{B}+1$, zero caso contrário; $D U_{t}=1$ se $t>T_{B}$, zero caso contrário; $D T_{t}=t$ se $t>T_{B}$, zero caso contrário. Note que $T_{B}$ se refere ao ano da quebra estrutural, isto é, o período no qual ocorre a mudança nos parâmetros da função de tendência. ${ }^{18}$

\footnotetext{
${ }^{16}$ A escolha de $n$ na equação de teste foi baseada no procedimento $t-s i g$, no nível de $10 \%$ de significância (Perron, 1997). A hipótese de resíduos não correlacionados foi testada utilizando-se a estatística-Q de Ljung e Box, sendo o número de autocorrelações adotado para obter-se esta estatística dado por $\underset{T}{\operatorname{Min}}\{T / 2,3 \sqrt{T}\}$, em que $T$ é o tamanho da amostra.

${ }^{17} \mathrm{Em}$ conseqüência do uso de diferenças defasadas nas equações de teste duas observações foram "perdidas", logo todos os testes de raiz unitaria abrangerão o período 1949-1999.

${ }^{18}$ Quanto à escolha do parâmetro $k$, para cada uma das equações acima, foi adotado o procedimento t-sig (Perron, 1997) a 10\% de significância.
} 
A determinação de $T_{B}$ é feita endogenamente, adotando-se um procedimento seqüencial que consiste na estimação da equação de teste para todos $T_{B}=k_{0}, \cdots$ $\cdot, T-k_{0}$, em que $k_{0}=\xi T$, sendo que $T$ é o tamanho da amostra e $\xi$ é um parâmetro de trimming, no caso igual a 5\%. Para cada uma das estimações da equação acima o "pseudo-t" $\left(\tau_{\tau}\right)$ para o coeficiente de $C_{t-1}$ é obtido, sendo o ponto de quebra determinado pela estatística seqüencial $\tau_{\text {seq }}$, tal que $\tau_{\text {seq }}=\min \tau_{\tau}$.

Ao aplicar este teste, obtivemos que o valor mínimo da estatística $\tau_{\text {seq }}$ corresponde ao ano de 1971 para a série CF e 1970 para a série CT. ${ }^{19}$ Em ambos os casos a hipótese nula de raiz unitária não é rejeitada, no nível de significância de $5 \%$ (tabelas A3 e A4).

Portanto, mesmo com uma equação de teste mais ampla, que permite variações na função de tendência, não foi encontrada evidência na amostra contra a hipótese nula de raiz unitária para as séries $\mathrm{CF}$ e $\mathrm{CT}$.

Como estimamos o consumo $I(1)$, concluímos que tal série é composta por um passeio aleatório e possivelmente por uma parte cíclica da seguinte forma:

$$
\begin{gathered}
c_{t}=c_{t}^{p}+c_{t}^{c} \\
c_{t}^{p}=c_{t-1}^{p}+\psi_{t} \\
\psi_{t} \quad \text { é uma inovação } \\
c_{t}^{c} \quad \text { é estacionário }
\end{gathered}
$$

Seja $w_{t}=c_{t}-c_{t-1}$. Este processo é estacionário e pelo teorema de decomposição de Wold pode ser representado por um $M A(\infty), w_{t}=\mu+\epsilon_{t}+\sum_{i=1}^{\infty} \lambda_{i} \epsilon_{t-i}$. Beveridge e Nelson mostraram que $c_{t}^{c}=\left(\sum_{i=1}^{\infty} \lambda_{i}\right) \epsilon_{t}+\left(\sum_{i=2}^{\infty} \lambda_{i}\right) \epsilon_{t-1}+\left(\sum_{i=3}^{\infty} \lambda_{i}\right)$ $\epsilon_{t-2}+\ldots$, de modo que, estimando um modelo $\operatorname{ARMA}(p, q)$ para $w_{t}$ e invertendo a parte $\mathrm{AR}$, temos o $M A(\infty)$ e os valores de $\lambda_{i}, i=1,2, \ldots$, podendo ser calculada a parte cíclica do consumo, se esta existir. ${ }^{20}$ Portanto, a existência de um ciclo

\footnotetext{
${ }^{19} \mathrm{O}$ ano $T_{B}$ deve ser visto apenas como uma aproximação do ano no qual ocorre a mudança estrutural na série do consumo. Isto porque o procedimento seqüencial visa escolher o ano que dá a maior probabilidade de se rejeitar a nula, ao invés de escolher o ano que maximiza a ocorrência de uma mudança na função de tendência, o que se justifica pelo fato deste teste pretender apenas estimar o grau de integração da série do consumo na presença de uma mudança no comportamento da série, não havendo prioridade em se determinar o ano desta possível mudança.

${ }^{20}$ Note que $w_{t}$ é estacionário. Logo se existir a parte AR, ela é invertível.
} 
depende fundamentalmente de $p \neq 0$ e/ou $q \neq 0$. Recordamos que a TRP implica $\lambda_{i}=0, i=1,2, \ldots$, dado que a primeira diferença de um passeio aleatório é $\epsilon_{t}$.

As séries CF e CT apresentam grande semelhança de modo que, doravante, utilizaremos apenas a primeira já que esta tem maior apelo teórico e o ganho de informação, incluindo a série CT é pequeno. Desta forma, a fim de implementar esta metodologia construímos a série $h_{t}=C F_{t}-C F_{t-1}$. Para esta série foi possível estimar alguns modelos do tipo $\operatorname{ARMA}(p, q)$, sendo o mais parcimonioso o $\operatorname{ARMA}(1,1), h_{t}=\phi h_{t-1}+\epsilon_{t}+\theta \epsilon_{t-1}$, cujos resultados são reportado abaixo na tabela 1.

Tabela 1

Estimação de $h_{t} \sim \operatorname{ARMA}(1,1)$

\begin{tabular}{cccc}
\hline Coeficiente & Estimativa & Estatística $t$ & $\mathrm{p}$-valor \\
\hline$\phi$ & 0,8978 & 5,5579 & 0,0000 \\
$\theta$ & $-0,7777$ & $-3,5247$ & 0,0009 \\
\hline \multicolumn{2}{l}{ Teste F: $\phi=\theta=0 \Rightarrow$ Estatística $F=22,05$ (p-valor $=0,0000)$} \\
\hline
\end{tabular}

$$
\begin{gathered}
M A(\infty): h_{t}=\epsilon_{t}+\sum_{i=1}^{\infty}\left[\phi^{i}\left(1+\frac{\theta}{\phi}\right) L^{i}\right] \epsilon_{t} \\
\text { Ciclo : } C F_{t}^{c}=\frac{(\phi+\theta)}{1-\phi} \sum_{i=0}^{\infty} \phi^{i} \epsilon_{t-i}
\end{gathered}
$$

Como $p=1$ e $q=1$, foi estimado um componente cíclico para a série CF, rejeitando-se a TRP. Esta rejeição não nos surpreende, dado o resultado de Reis et alii (1998). De fato, ao utilizarmos as séries de CF e PIB e a equação (2) obtemos uma estimativa de $\lambda$ que reproduz o resultado de Reis et alii (1998), reportada na tabela $2 .^{21}$

\footnotetext{
${ }^{21}$ No apêndice B é apresentada a análise do grau de integração do PIB. Ao contrário de Cati (1998), concluí-se que tal série é $I(1)$. Assim, $\Delta$ PIB bem como $\Delta$ CF são $I(0)$, estando a regressão entre ambos bem especificada.
} 
Tabela 2

Estimação do modelo: $\Delta C_{t}=\lambda \Delta Y_{t}+\epsilon_{t}$

\begin{tabular}{cccc}
\hline \multicolumn{2}{l}{ Período $1950-1999$} & & \\
\hline Linha & Instrumentos & $\lambda$ & $\begin{array}{c}\text { Teste de } \\
\text { Sobreidentificação }\end{array}$ \\
\hline 1 & $\Delta C_{t-2}, \Delta Y_{t-2}$ & 0,9669 & 2,4601 \\
& & $-11,0756$ & $-3,841$ \\
\hline 2 & $\Delta C_{t-2}, \Delta Y_{t-2}$, & 0,9778 & 2,6072 \\
& $\Delta C_{t-3}, \Delta Y_{t-3}$ & $-11,0567$ & $-7,815$ \\
\hline
\end{tabular}

Nota: As variáveis $C$ e $Y$ referem-se às séries CF e PIB, respectivamente. A terceira coluna apresenta entre parênteses a estatística $t$ e a última apresenta o valor crítico da distribuição $\chi^{2}$ a $5 \%$ de significância.

Estimamos $\lambda$ próximo de um e estatisticamente diferente de zero, no nível de significância de 5\%. A partir da decomposição de Beveridge e Nelson do consumo surge uma possível explicação para este resultado, que é o fato de que a série de consumo agregado possui um ciclo não contemplado pela TRP (Hall, 1978, Flavin, 1981). Logo, ao estimar a equação de teste acima, $\Delta Y_{t}$ torna-se uma proxy para tal ciclo e por isso estima-se $\lambda$ estatisticamente diferente de zero. Sob este raciocínio, ao se levar em conta na equação de teste um processo para o consumo que possua um ciclo, a estimativa de $\lambda$ não deve permanecer significativamente diferente de zero. Para avaliar inclusive esta questão consideraremos o modelo com formação de hábito através da equação de teste (3). Os resultados são reportados na tabela 3 abaixo.

Tabela 3

Estimação do modelo: $\Delta C_{t}=\lambda \Delta Y_{t}+(1-\lambda)\left(\beta_{1} \Delta C_{t-1}+\beta_{2} \Delta C_{t-2}\right)+\mu_{t}$

\begin{tabular}{cccccc}
\hline \multicolumn{2}{l}{ Período } & 1951-1999 & & & \\
\hline Linha & Instrumento & $\lambda$ & $(1-\lambda) \beta_{1}$ & $(1-\lambda) \beta_{2}$ & $\begin{array}{c}\text { Teste de } \\
\text { Sobreidentificação }\end{array}$ \\
\hline 1 & $\Delta C_{t-2}, \Delta Y_{t-2}$ & 1,1112 & 0,0296 & $-0,1492$ & 0,5484 \\
& $\Delta C_{t-3}, \Delta Y_{t-3}$ & $-3,8909$ & $-0,1353$ & $(-1,179)$ & $-3,8415$ \\
\hline 2 & $\Delta C_{t-2}, \Delta Y_{t-2}$ & 1,1627 & $-0,0357$ & $-0,1504$ & 3,1872 \\
& $\Delta C_{t-3}, \Delta Y_{t-3}$ & $-4,1974$ & $(-0,1691)$ & $(-1,2028)$ & $-7,8147$ \\
& $\Delta C_{t-3}, \Delta Y_{t-3}$ & & & & \\
\hline
\end{tabular}

Nota: As variáveis $C$ e $Y$ referem-se às séries $\mathrm{CF}$ e PIB, respectivamente. Com exceção da última coluna, que reporta entre parênteses o valor crítico da distribuição $\chi^{2}$ a $5 \%$ de significância, as demais contem a estatística $t$ entre parênteses.

Novamente obtivemos $\lambda$ significativamente diferente de zero, no nível de significância de $5 \%$. Além disso, as estimativas de $(1-\lambda) \beta_{1}$ e $(1-\lambda) \beta_{2}$ são não signifi- 
cativas, no nível de significância de 5\%, indicando que, ao contrário do postulado, $\Delta Y_{t}$ não é uma proxy para um componente cíclico auto-regressivo do consumo, mas antes há realmente um excesso de sensibilidade do consumo à renda. Isto nos permite concluir ainda que a existência de um ciclo no consumo não se deve a formação de hábito, mas ao fato de os agentes estarem restritos a consumir sua renda corrente.

Resta investigar se o resultado acima se deve ao fato de termos omitido na equação de teste um termo $\mathrm{MA}(1)$, pois ao realizar a decomposição de Beveridge e Nelson do consumo estimamos $\Delta C_{t} \sim \operatorname{ARMA}(1,1)$. Inclusive a omissão do termo MA(1) pode estar causando o resultado não esperado sobre o valor estimado de $\lambda$. As estimativas para $\lambda$ quando se inclui um termo MA(1) são reportadas abaixo na tabela 4 .

Tabela 4

Estimação do modelo: $\Delta C_{t}=\lambda \Delta Y_{t}+(1-\lambda)\left(\beta_{1} \Delta C_{t-1}+\beta_{2} \Delta C_{t-2}\right)+\delta \epsilon_{t-1}+\epsilon_{t}$

\begin{tabular}{ccccccc}
\hline Período $1952-1999$ & \multicolumn{5}{c}{} & \\
\hline Linha & Instrumento & $\lambda$ & $(1-\lambda) \beta_{1}$ & $(1-\lambda) \beta_{2}$ & $\delta$ & $\begin{array}{c}\text { Teste de } \\
\text { Sobreidentificação }\end{array}$ \\
\hline 1 & $\Delta C_{t-2}, \Delta Y_{t-2}$ & 0,8197 & 0,2099 & $-0,0704$ & $-0,7911$ & 2,2589 \\
& $\Delta C_{t-3}, \Delta Y_{t-3}$ & $-4,4323$ & $-1,497$ & $-0,5953)$ & $(-5,6269)$ & $-5,9915$ \\
& $\Delta C_{t-4}, \Delta Y_{t-4}$ & & & & & \\
\hline 2 & $\Delta C_{t-2}, \Delta Y_{t-2}$ & 0,8842 & 0,1487 & $-0,0702$ & $-0,7266$ & 5,3615 \\
& $\Delta C_{t-3}, \Delta Y_{t-3}$ & $-4,6481$ & $-1,0515$ & $(-0,6095)$ & $(-4,839)$ & $-9,4877$ \\
& $\Delta C_{t-4}, \Delta Y_{t-4}$ & & & & & \\
& $\Delta C_{t-5}, \Delta Y_{t-5}$ & & & & & \\
\hline
\end{tabular}

Nota: As variáveis $C$ e $Y$ referem-se às séries $\mathrm{CF}$ e $\mathrm{PIB}$, respectivamente.

Com exceção da última coluna, que reporta entre parênteses o valor

crítico da distribuição $\chi^{2}$ a $5 \%$ de significância, as demais contem a

estatística $t$ entre parênteses.

Novamente $\lambda$ é significativamente diferente de zero, no nível de significância de $5 \%$, não se podendo rejeitar a hipótese de que as variações na renda desempenham papel crucial na explicação das variações no consumo. Mais uma vez, as estimativas de $(1-\lambda) \beta_{1}$ e $(1-\lambda) \beta_{2}$ são não significativas. Por fim, cabe ressaltar que não há um ciclo comum entre consumo e renda, já que o coeficiente $\delta$ é significativamente diferente de zero, no nível de significância de 5\%. No entanto, não se descarta a hipótese de ambos possuírem um ciclo codependente (Vahid e Engle, 1997)..$^{22}$

\footnotetext{
${ }^{22}$ A existência de um ciclo codependente entre consumo e renda é sugerida devido a não rejeição do teste de sobreidentificação, no nível de significância de $5 \%$.
} 
Portanto, os resultados acima sugerem que o componente cíclico do consumo deve-se ao fato de os agentes seguirem a regra de bolso de consumir a sua renda corrente, o que sugere que grande parte da população brasileira não tem acesso ao crédito.

\section{Conclusões}

O presente artigo teve como objetivo estudar a série de consumo agregado no Brasil, de 1947 a 1999. Para tanto, investigou-se a aplicabilidade da TRP através da decomposição de Beveridge e Nelson. Segundo tal decomposição, esta série pode ser representada por dois componentes: um passeio aleatório e um ciclo estacionário. A TRP implica que não deveria existir uma parte cíclica no consumo, o que não é corroborado pelos dados. Portanto, explicitando-se o componente cíclico do consumo, rejeita-se a TRP.

A partir deste resultado, o presente trabalho incorporou preferências que exibem formação de hábito, sendo derivado a partir do problema de otimização do consumidor um processo estocástico para o consumo, contendo um componente cíclico, além da tendência estocástica. No entanto, este processo quando comparado à regra de consumir a renda corrente, é estatisticamente não significativo. Portanto, os resultados encontrados sugerem que a série temporal do consumo no Brasil é melhor explicada quando se considera um agente que segue a regra de bolso de consumir a sua renda corrente, possivelmente devido à falta de acesso ao crédito.

\section{Referências}

Banerjee, A. (1993). Co-Integration, Error-Correction, and the Econometric Analysis of Non-Stationary Data. Advanced Texts in Econometrics. Oxford University Press.

Beveridge, S. \& Nelson, C. R. (1981). A new approach to decomposition of economic time series into permanent and transitory components with particular attention to measurement of the business cycle. Journal of Monetary Economics, $7(2): 151-174$.

Campbell, J. \& Mankiw, G. (1989). Consumption, Income and Interest Rates: Reinterpreting the Time Series Evidence. MIT Press, Cambridge, MA. Olivier J. Blanchard \& Stanley Fischer. National Bureau of Economic Research Macroeconomics Annual. 
Cati, R. C. (1998). Stochastic and segmented trends in Brazilian GDP from 1900 to 1993. XX Encontro Brasileiro de Econometria. Vitória, Espírito Santo.

Engle, R. F. \& Kozicki, S. (1993). Testing for common features. Journal of Business and Economics Statistics, 11:369-395.

Flavin, M. (1981). The adjustment of consumption to changing expectations about future income. Journal of Political Economy, 89(5):974-1009.

Hall, R. E. (1978). Stochastic implications of the life cycle-permanent income hypothesis: Theory and evidence. Journal of Political Economy, 86:971-987.

Hamilton, J. (1994). Time Series Analysis. Princeton University Press, Princeton.

Holden, D. \& Perman, R. (1994). Unit roots and cointegration for the economist. In Rao, B. (org.), editor, Cointegration for the Applied Economist, pages 47-95. St. Martin's Press, Kensington, Australia.

IPEADATA (2000). http://www.ipeadata.gov.br/ipeaweb.dll.

Perron, P. (1989). The great crash, the oil price shock, and the unit root hipothesis. Econometrica, 57(6):1361--1401.

Perron, P. (1997). Further evidence on breaking trend functions in macroeconomic variables. Journal of Econometrics, 80(2):355- -385.

Rabin, M. (1998). Psychology and economics. Journal of Economic Literature, XXXVI:11-46.

Reis, E., Issler, J. V., Blanco, F., \& Carvalho, L. (1998). Renda permanente e poupança precaucional: Evidências empíricas para o Brasil no passado recente. Pesquisa e Planejamento Econômico, 28(2):233-272.

Rocha, F. \& Issler, J. V. (2000). Consumo, restrição à liquidez, e bem estar no Brasil. Economia Aplicada, 4(4).

Vahid, F. \& Engle, R. F. (1997). Codependent cycles. Journal of Econometrics, 80(2):199-121.

Weber, C. E. (2002). Intertemporal non-separability and "rule of thumb" consumption. Journal of Monetary Economics, 49(2):293-308. 


\section{Apendice A - Figuras e Tabelas}

Figura A.1

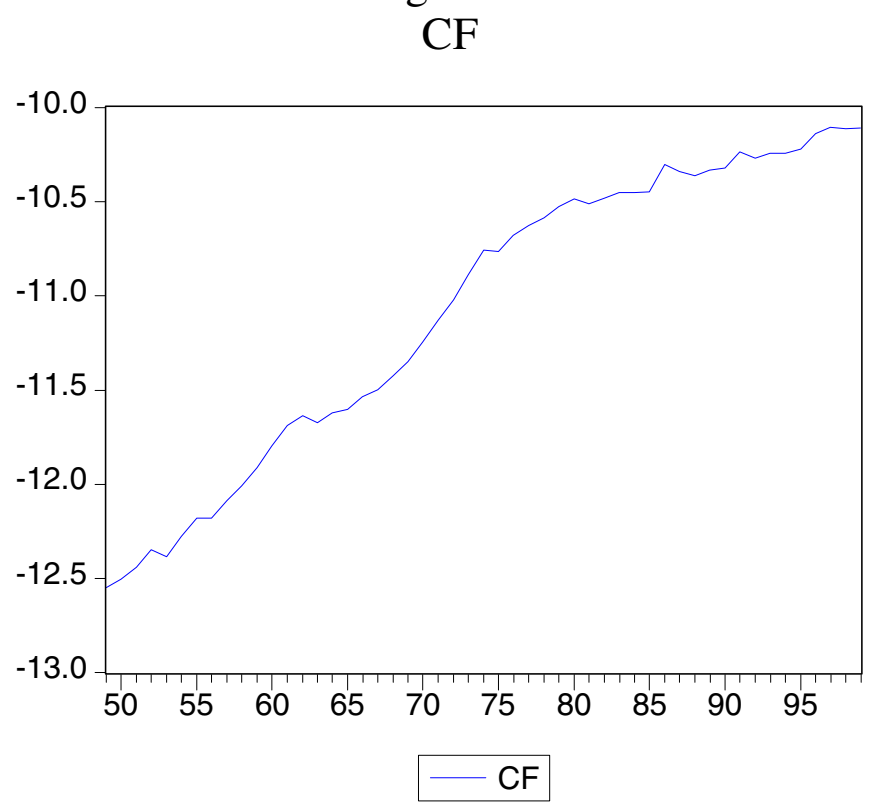

Figura A.2

$\mathrm{CF}$

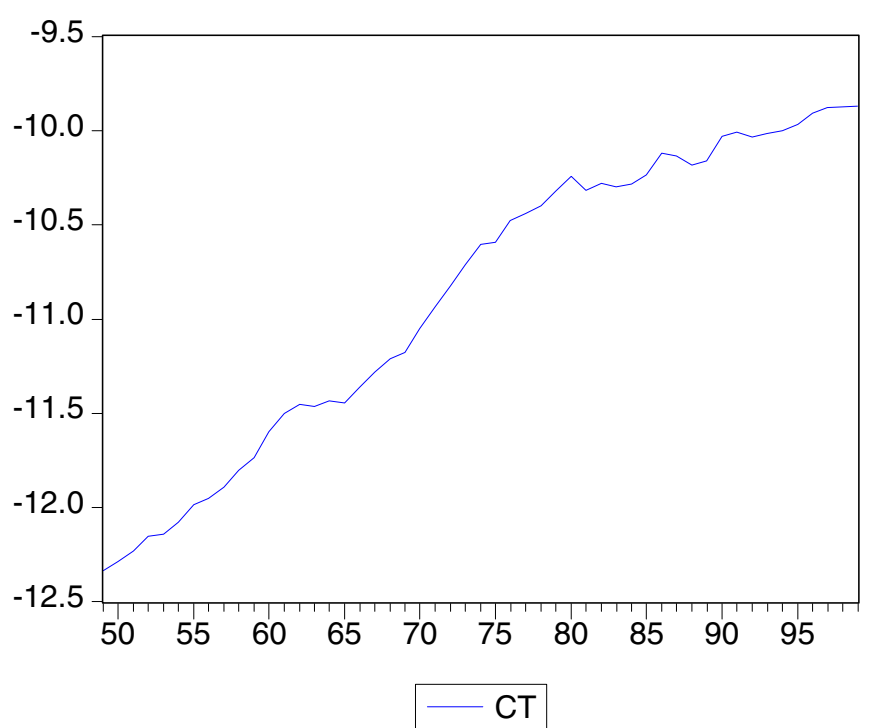


Figura A.3

PIB

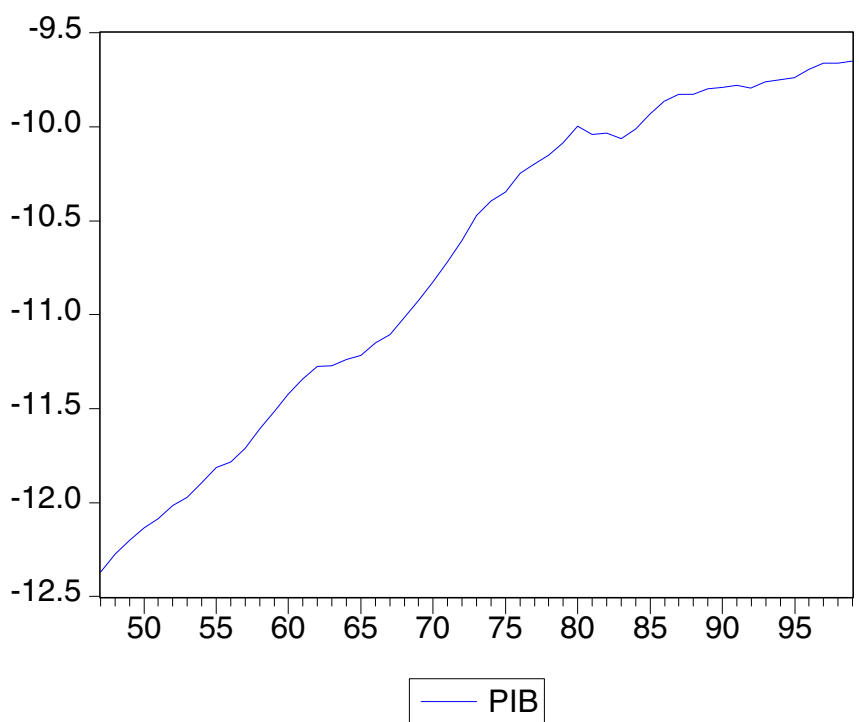

Figura A.4

Correlograma-CF
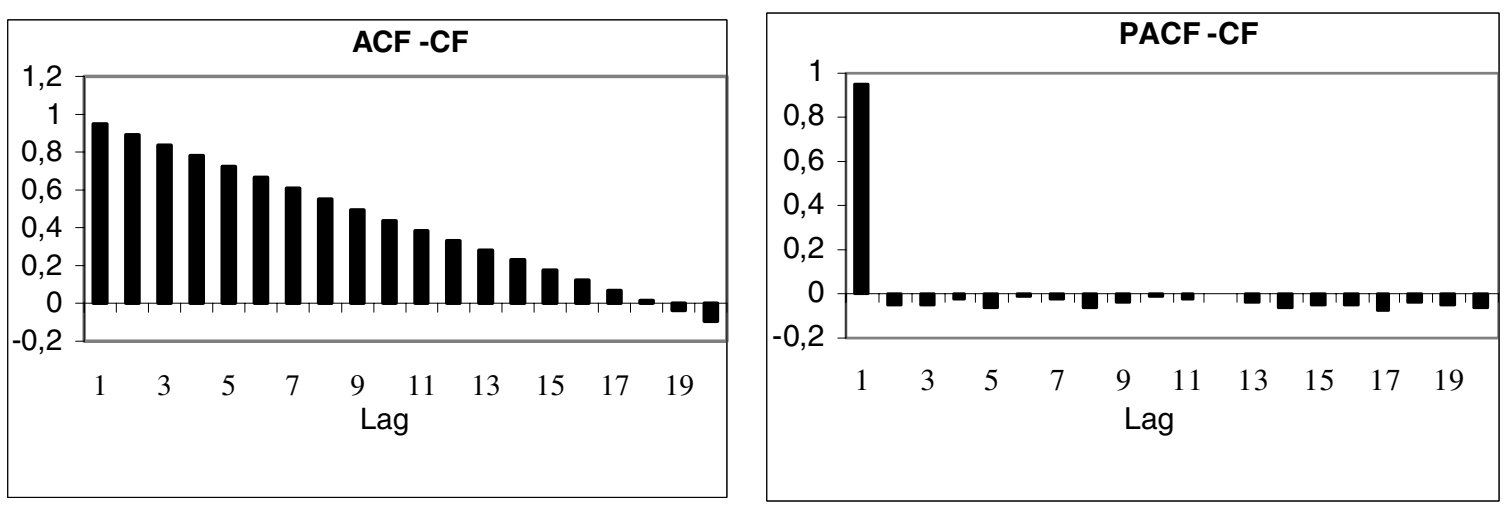
Figura A.5

Correlograma - CT
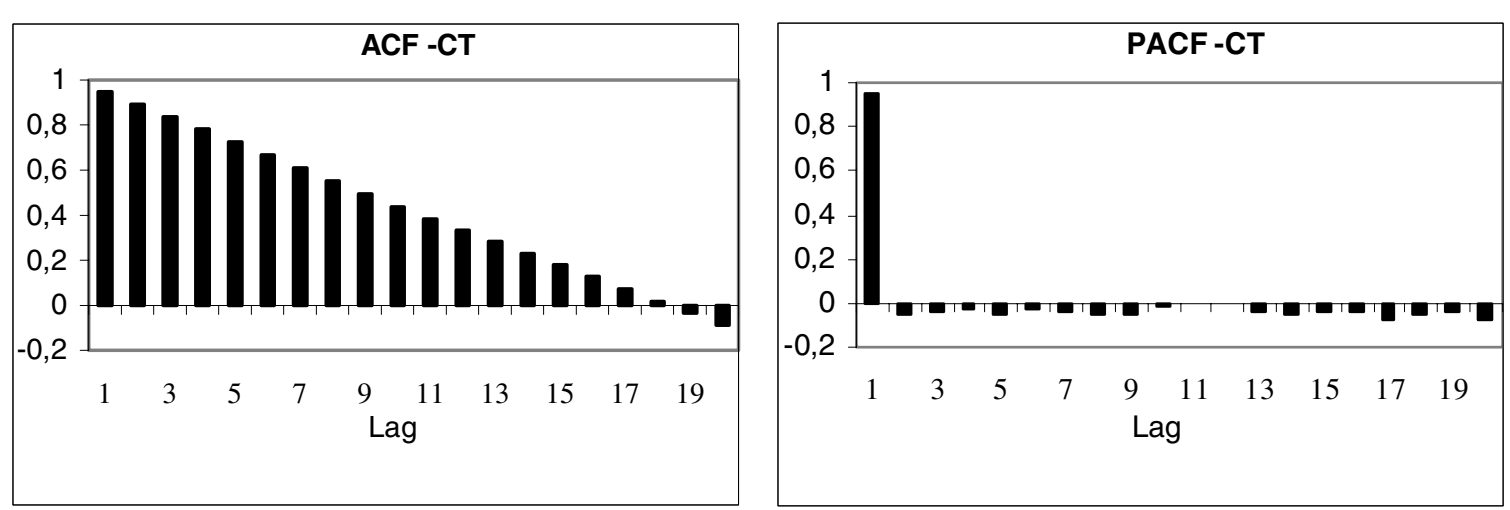

Figura A.6

Correlograma - PIB
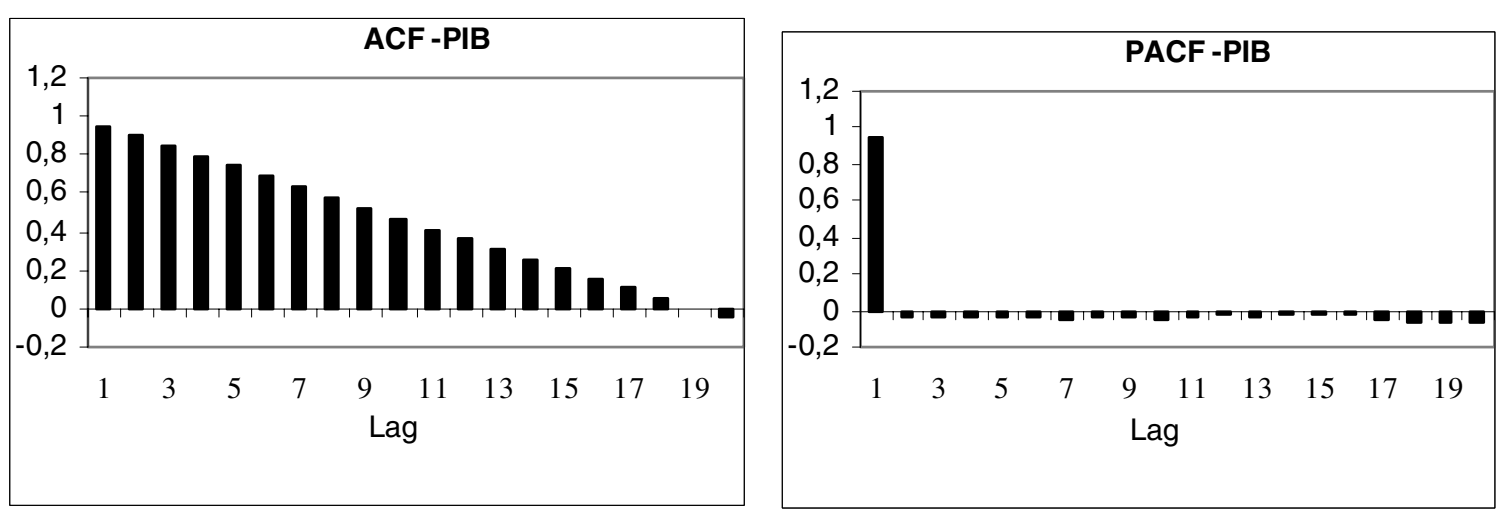
Tabela A.1 - Teste de R.U. para CF

\begin{tabular}{ccccccc}
\hline $\begin{array}{c}\text { Variável dependente: } \Delta C F_{t} \\
\text { Período: 1949-1999 }\end{array}$ & $\mathrm{N}$ & Intercepto & $\mathrm{CFt}-1$ & \multicolumn{3}{c}{ Valores Críticos } \\
\hline Modelo & & & & $1 \%$ & $5 \%$ & $10 \%$ \\
\hline Coeficiente & & -0.1913 & -0.0216 & & & \\
Desvio-padrão & 0 & 0.0911 & 0.0082 & -3.5625 & -2.9190 & -2.5970 \\
Estatística $t$ & & -2.0991 & -2.6461 & & & \\
\hline
\end{tabular}

Notas: Valores críticos de Mackinnon fornecidos pelo software Econometrics Views.

A amostra inclui 51 observações. "N" é o número de diferenças da variável dependente incluídas no lado direito da equação de teste.

O p-valor da estatística-Q de LB é 0.656.

Tabela A.2 - Teste de R.U. para $C T$

\begin{tabular}{ccccccc}
\hline $\begin{array}{c}\text { Variável dependente: } \Delta C T_{t} \\
\text { Período: 1949-1999 }\end{array}$ & & & & & \\
\hline Modelo & $\mathrm{N}$ & Intercepto & $C T_{t-1}$ & \multicolumn{3}{c}{ Valores Críticos } \\
\cline { 4 - 6 } & & & & $1 \%$ & $5 \%$ & $10 \%$ \\
\hline Coeficiente & & -0.1713 & -0.0202 & & & \\
Desvio-padrão & 0 & 0.0863 & 0.0078 & -3.5625 & -2.9190 & -2.5970 \\
Estatística $t$ & & -1.9836 & -2.5701 & & & \\
\hline
\end{tabular}

Notas: Valores críticos de Mackinnon fornecidos pelo software Econometrics Views.

A amostra inclui 51 observações. "N " é o número de diferenças da variável dependente incluídas no lado direito da equação de teste.

O p-valor da estatística-Q de LB é 0.174 .

Tabela A.3 -Teste de RU (Perron, 1989) para o $C F$

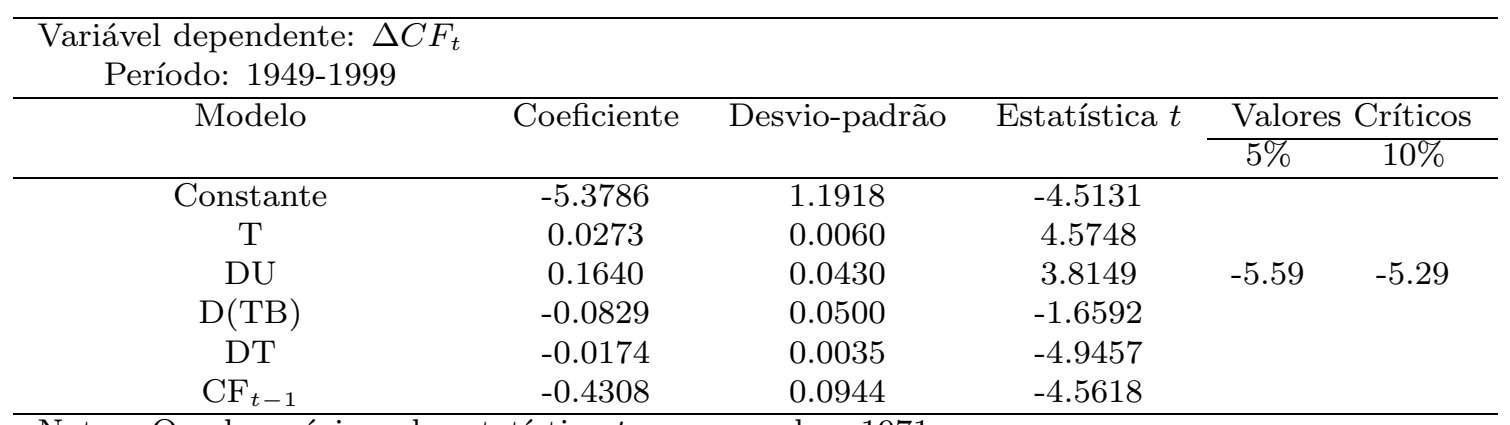

Notas: O valor mínimo da estetística $t$ corresponde a 1971 .

Valores críticos de Perron (1997) para $T=70$.

A amostra inclui 51 observações. O p-valor da estatística-Q de LB é 0.645. 
Tabela A.4 - Teste de RU Perron (1989) para o $C T$

\begin{tabular}{|c|c|c|c|c|c|}
\hline \multicolumn{6}{|c|}{$\begin{array}{l}\text { Variável dependente: } \Delta C T_{t} \\
\text { Período: } 1949-1999\end{array}$} \\
\hline \multirow[t]{2}{*}{ Modelo } & \multirow{2}{*}{ Coeficiente } & \multirow{2}{*}{ Desvio-padrão } & \multirow[t]{2}{*}{ Estatística $t$} & \multicolumn{2}{|c|}{ Valores Críticos } \\
\hline & & & & $5 \%$ & $10 \%$ \\
\hline Constante & -4.1192 & 1.0223 & -4.0288 & & \\
\hline $\mathrm{T}$ & 0.0205 & 0.0051 & 3.9744 & & \\
\hline DU & 0.1360 & 0.0376 & 3.6194 & -5.59 & -5.29 \\
\hline $\mathrm{D}(\mathrm{TB})$ & -0.0657 & 0.0490 & -1.3439 & & \\
\hline $\mathrm{DT}$ & -0.0118 & 0.0028 & -4.2766 & & \\
\hline $\mathrm{CT}_{t-1}$ & -0.3370 & 0.0824 & -4.0893 & & \\
\hline
\end{tabular}

Notas: O valor mínimo da estetística $t$ corresponde a 1970.

Valores críticos de Perron (1997) para $T=70$.

A amostra inclui 51 observações. O p-valor da estatística-Q de LB é 0.078 . 
